\title{
Epinephrine during CPR significantly increases organ perfusion in a rat VF cardiac arrest model
}

Raphael Schemm, Teresa Berger, Christian Clodi, Christoph Schriefl, Wolfgang Weihs, Michael Holzer, Alexandra Warenits, Andreas Schober, Ingrid Magnet, Florian Ettl

Medical University of Vienna - Department of Emergency Medicine,

- Epinephrine

- No Epinephrine

Währinger Gürtel 18-20, 1090 Vienna, Austria

\section{INTRODUCTION}

Epinephrine is an established vasopressor and the primary drug administrated during cardiopulmonary resuscitation (CPR). This study evaluates the influence of epinephrine on coronary perfusion pressure (CPP), mean atrial pressure (MAP) and end-tidal $\mathrm{CO} 2$ (ETCO2) in our rat VF CPR model.

\section{METHODS}

Adult male Sprague-Dawley rats $(400 \mathrm{~g})$ were randomized to CPR with $(n=15)$ and without epinephrine $(n=10)$. After 6 minutes of untreated cardiac arrest (CA) study animals were resuscitated with mechanical chest compressions, ventilations and defibrillations. In the epinephrine group, epinephrine 10 $\mu \mathrm{g} / \mathrm{kg}$ were administered every two minutes.

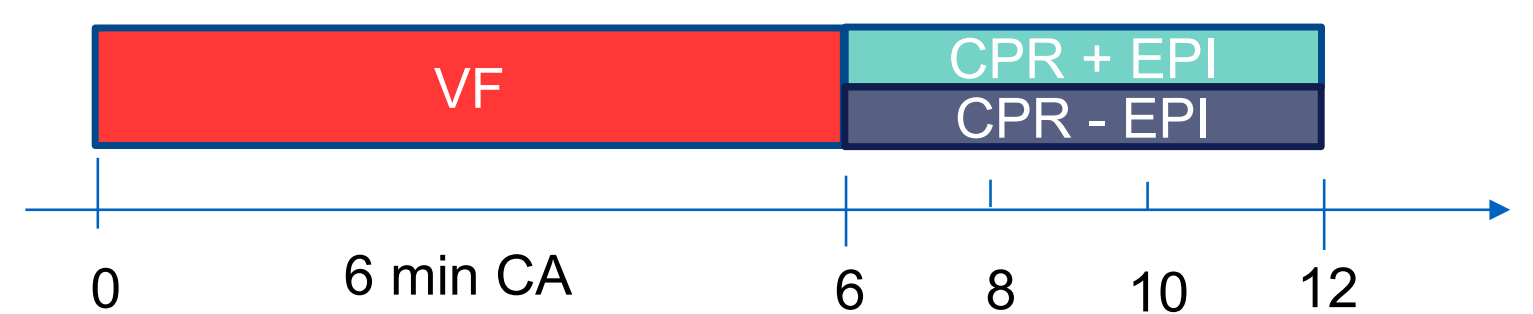

Mean arterial pressure (MAP), central venous pressure (CVP) and ETCO2 were continuously monitored and calculated for the first two minutes of CPR. Mean CPP was calculated as difference between MAP and CVP.

\section{RESULTS}

The mean CPP was significantly higher during CPR in animals resuscitated with epinephrine than without epinephrine. The epinephrine group showed also significantly higher MAP values. The mean ETCO2 was higher in the no epinephrine group.

\begin{tabular}{|lcc|}
\hline [mmHg] & Epinephrine & No Epinephrine \\
\hline CPP & $38 \pm 8$ & $23 \pm 4$ \\
\hline MAP & $45 \pm 8$ & $25 \pm 6$ \\
\hline etCO2 & $32 \pm 6$ & $42 \pm 8$ \\
\hline
\end{tabular}

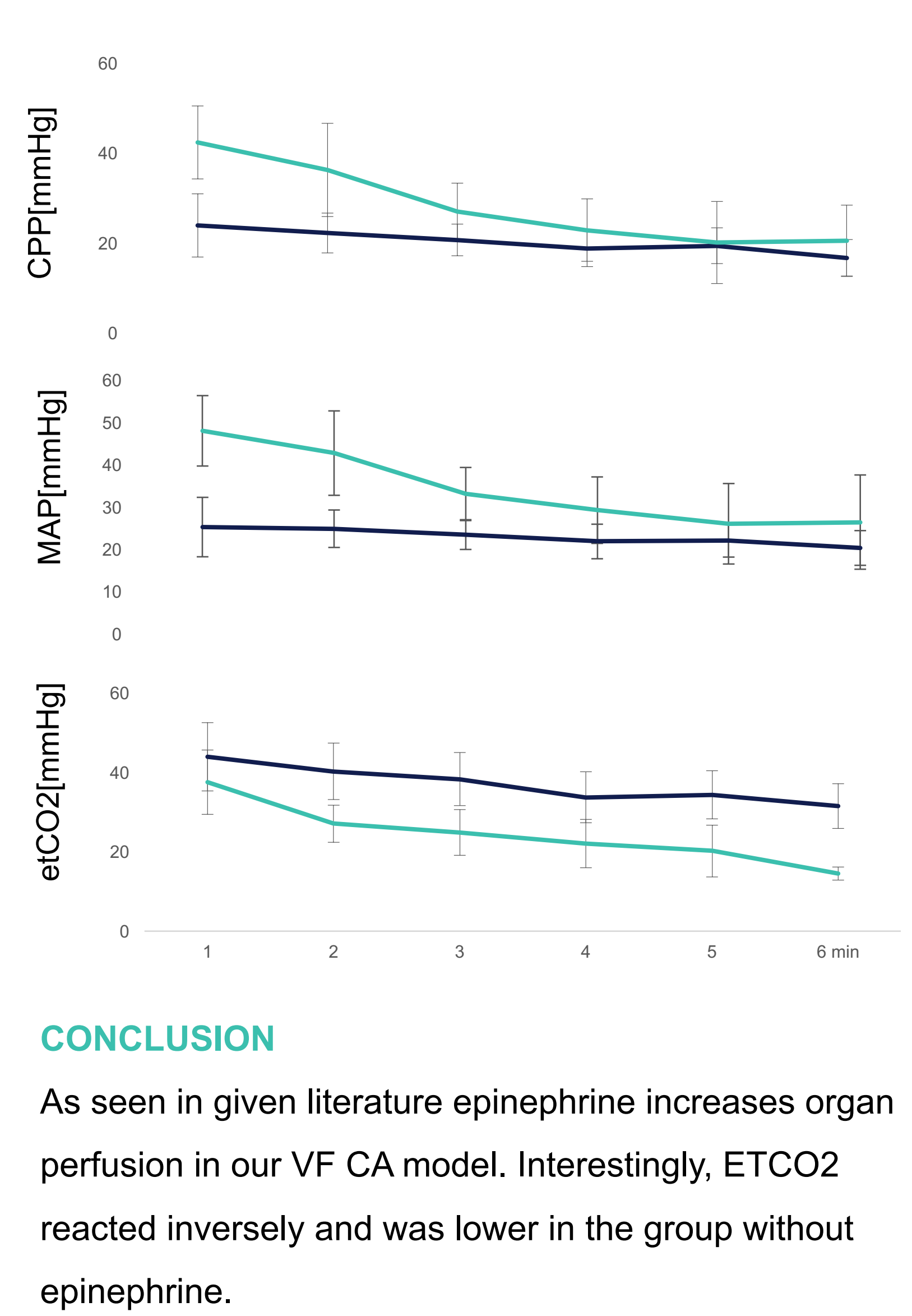

